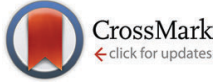

Cite this: Phys. Chem. Chem. Phys., 2015, 17, 4864

Received 10th December 2014, Accepted 14th January 2015

DOI: $10.1039 / c 4 c p 05775 a$

www.rsc.org/pccp

\section{The critical role of intragap states in the energy transfer from gold nanoparticles to $\mathrm{TiO}_{2} \dagger$}

\author{
Alberto Naldoni, ${ }^{{ }^{a}}$ Filippo Fabbri, ${ }^{\mathrm{b}}$ Marco Altomare, $\ddagger^{\mathrm{C}}$ Marcello Marelli, ${ }^{a}$ \\ Rinaldo Psaro, ${ }^{a}$ Elena Selli, ${ }^{c}$ Giancarlo Salviati ${ }^{\mathrm{b}}$ and Vladimiro Dal Santo*a
}

\begin{abstract}
Cathodoluminescence spectroscopy is profitably exploited to study energy transfer mechanisms in $\mathrm{Au}$ and $\mathrm{Pt} /$ black $\mathrm{TiO}_{2}$ heterostructures. While Pt nanoparticles absorb light in the UV region, Au nanoparticles absorb light by surface plasmon resonance and interband transitions, both of them occurring in the visible region. The intra-bandgap states (oxygen vacancies) of black $\mathrm{TiO}_{2}$ play a key role in promoting both hot electron transfer and plasmonic resonant energy transfer from $\mathrm{Au}$ nanoparticles to the $\mathrm{TiO}_{2}$ semiconductor with a consequent photocatalytic $\mathrm{H}_{2}$ production increase. An innovative criterion is introduced for the design of plasmonic composites with increased efficiency under visible light.
\end{abstract}

\section{Introduction}

The urgent need to provide methods for efficient conversion of solar light into electricity (i.e., photovoltaics) and solar fuels (i.e., photocatalysis and photo-electrochemistry) has triggered the development of more effective approaches to exploit semiconductor metal oxides.

In particular, the progress rapidly gained in the field of plasmonics has paved the way for the fabrication of devices with enhanced efficiency. ${ }^{1-4}$ For instance, the modification of semiconductors with metals (e.g., Au or Ag nanostructures) was shown to increase the efficiency of solar cells by $10-15 \%$ and to improve the photocatalytic activity in both water splitting reactions and decomposition of organic molecules. ${ }^{4}$

Besides the physical benefits derived from the use of metal nanostructures, such as the extension of visible light absorption and light scattering amplification, plasmonic interactions (PIs)

\footnotetext{
${ }^{a}$ CNR-Istituto di Scienze e Tecnologie Molecolari, Milano, Italy.

E-mail: a.naldoni@istm.cnr.it,v.dalsanto@istm.cnr.it

${ }^{b}$ IMEM-CNR, Parma, Italy

${ }^{c}$ Università degli Studi di Milano, Dipartimento di Chimica, Milano, Italy

$\dagger$ Electronic supplementary information (ESI) available: Details of synthesis, experimental methods, additional materials characterization and photocatalytic methanol steam reforming data. See DOI: 10.1039/c4cp05775a

\# Current address: Friedrich-Alexander University, Department of Materials Science Institute for Surface Science and Corrosion (LKO), Erlangen, Germany.
}

such as electron and energy transfer from the surface plasmon resonance (SPR) of metals to the semiconductor electronic structure play a major role. ${ }^{2,3}$ In addition, recent reports have shown that gold interband transitions (more energetic than plasmons, i.e. $E_{\text {interband }}=2.5 \mathrm{eV}$ ) may also have a significant role in below bandgap sensitization of semiconductors. ${ }^{5,6}$

Hot electron transfer (plasmonic or due to interband transitions) from metals to the conduction band (CB) of several metal oxides $\left(\mathrm{SrTiO}_{3},{ }^{5} \mathrm{TiO}_{2},{ }^{7-9} \mathrm{CeO}_{2},{ }^{10,11}\right.$ and $\left.\mathrm{ZnO}^{12}\right)$ has been observed and accounted for their increased photoactivity. In addition, plasmonic resonant energy transfer (PRET) can occur from the electromagnetic field generated at the surface of metal nanostructures to semiconductors having a bandgap resonant with the SPR band (i.e., $\alpha-\mathrm{Fe}_{2} \mathrm{O}_{3}, \mathrm{Cu}_{2} \mathrm{O}$, and $\mathrm{N}$-doped $\left.\mathrm{TiO}_{2}\right) .{ }^{13-19}$ PRET allows increased separation of photogenerated charges at the semiconductor/ metal interface, thus being a powerful tool toward the engineering of a novel generation of optoelectronic devices.

Considering wide-bandgap semiconductors (i.e., $\mathrm{TiO}_{2}$ ), the presence of intragap energy levels related to defects can play a significant role in PRET promotion. For example, water splitting photoanodes based on $\mathrm{N}$-doped $\mathrm{TiO}_{2}$ showed enhanced photocurrent behavior induced by PRET. ${ }^{17,18} \mathrm{~N}$-doping introduces intrabandgap levels above the $\mathrm{TiO}_{2}$ valence band that can resonate with the electromagnetic field, generated by SPR, propagating from the metal surface. The electromagnetic field is then able to promote charge carrier generation from $\mathrm{N}$-related levels to the $\mathrm{TiO}_{2}$ conduction band (where they participate in proton reduction), thus increasing the photoconversion efficiency. ${ }^{17,18}$ Nevertheless, $\mathrm{N}$-doping is quite specific for $\mathrm{TiO}_{2}$ and cannot be considered a design criterion extendible to a large family of oxides.

On the other hand, oxides in their native form contain point defects (e.g., oxygen vacancies and interstitial metal atoms) that profoundly influence their properties. In this regard, oxygen vacancies $\left(\mathrm{V}_{\mathrm{O}} \mathrm{S}\right)$ are most interesting; their concentration tuning ${ }^{20}$ can be exploited in a variety of functional materials ranging from photocatalysts, ${ }^{21}$ electrodes for fuel cells ${ }^{22}$ and Li-ion batteries ${ }^{23}$ to strongly-correlated materials (e.g., cuprates, manganites, and cobaltites) used for their superconductivity or magneto-resistive 
behavior. ${ }^{24}$ Moreover, $\mathrm{V}_{\mathrm{O}}$ s-related electronic levels, lying below the $\mathrm{CB}$, can provide relevant interactions with additional charges generated by excitation of metal nanoparticles (NPs).

However, to the best of our knowledge, despite the relevance of $\mathrm{V}_{\mathrm{O}} \mathrm{s}$, their role in hot electron transfer and in PRET has never been studied before. In this work we investigate the interactions between the plasmonic modes and interband transitions of $\mathrm{Au}$ nanoparticles and the electronic structure of black $\mathrm{TiO}_{2}{ }^{21}$ by means of cathodoluminescence (CL) spectroscopy. ${ }^{25-27}$ The aim of the study is twofold: on the one hand we highlight the effect of $\mathrm{V}_{\mathrm{O}} \mathrm{S}$ intragap states on the energy transfer (electron and energy) from $\mathrm{Au}$ NPs to $\mathrm{TiO}_{2}$; on the other hand we discern the contribution of carrier generated plasmons and gold interband transition excitation to photoacatalytic hydrogen production by methanol photo-steam reforming.

\section{Results and discussion}

Fig. 1 shows the morphological features of a single core-shell black $\mathrm{TiO}_{2} \mathrm{NP}$ - the defective crystalline core (Fig. 1a and b), and the disordered, i.e., amorphous, yet stoichiometric shell (Fig. 1a and c). ${ }^{21}$ The latter has a thickness of $1.5 \mathrm{~nm}$, as shown by the line profile obtained by the HRTEM image shown in Fig. 1d. The lattice distance for black $\mathrm{TiO}_{2}$ is $0.343 \mathrm{~nm}$ (Fig. 1d), while for P25 it corresponds to $0.347 \mathrm{~nm}$. Such values can be assigned to the anatase (101) reflection since the reciprocal angle between the (101) and (004) reflections (the FFT image reported in Fig. 1b) is $67^{\circ}$. The contraction of the (101) lattice distance highlights that the introduction of $\mathrm{V}_{\mathrm{O}} \mathrm{S}$ in the $\mathrm{TiO}_{2}$ lattice induces a reduction of the interplanar distance at the local scale. ${ }^{21}$ It is interesting to note that differently from black $\mathrm{TiO}_{2}$ (Fig. 1a) the boundaries of the P25 crystallite (Fig. 1e) are very well defined, showing the morphological difference between P25 (reference) and black $\mathrm{TiO}_{2}$. The straightforward reduction of the commercial amorphous $\mathrm{TiO}_{2}$ produces a highly defective material, containing $c a$. $5 \%$ of $\mathrm{V}_{\mathrm{O}} \mathrm{S}$ and showing an intense absorption of visible light. ${ }^{21}$

Black $\mathrm{TiO}_{2}$ NPs were modified with Au and Pt NPs through a wet deposition procedure described elsewhere. ${ }^{28}$ We chose to deposit Pt and $\mathrm{Au}$ in order to compare NPs showing SPR in different energy ranges. In fact, Pt SPR peaks above the $\mathrm{TiO}_{2}$ bandgap, while Au plasmon can resonate with intragap states of defective titania. SPR of Pt falls in the UV rather than in the visible region and it is usually difficult to observe because it overlaps with the intense oxide bandgap absorption. Pt does not have the optimum cross section and non-radiative plasmon deactivation efficiency shown by Au or Ag. Nevertheless, the Pt SPR was observed in many different nanostructures. ${ }^{29-33}$

We first focus on samples modified with Au NPs. TEM and STEM images of Au/P25 (Fig. 2a and b) and Au/black $\mathrm{TiO}_{2}$ (Fig. 2f) show that the metallic NPs have a homogeneous size and are well dispersed over the surface of the semiconductor. In particular, high resolution TEM images (Fig. 2e) clearly show that gold nanoparticles form an interface only with the amorphous layers of black $\mathrm{TiO}_{2}$, and do not come into contact with the

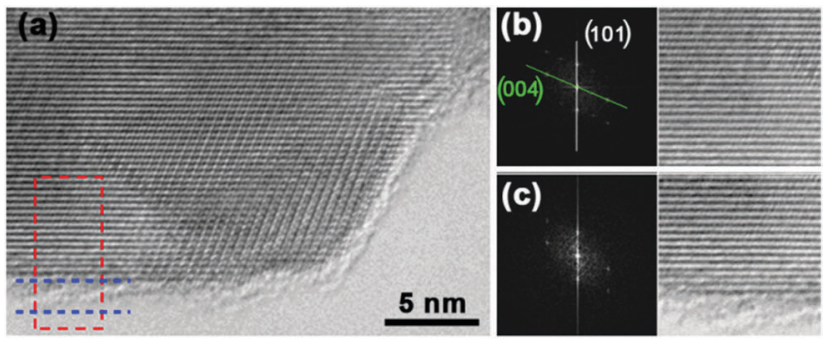

(d)
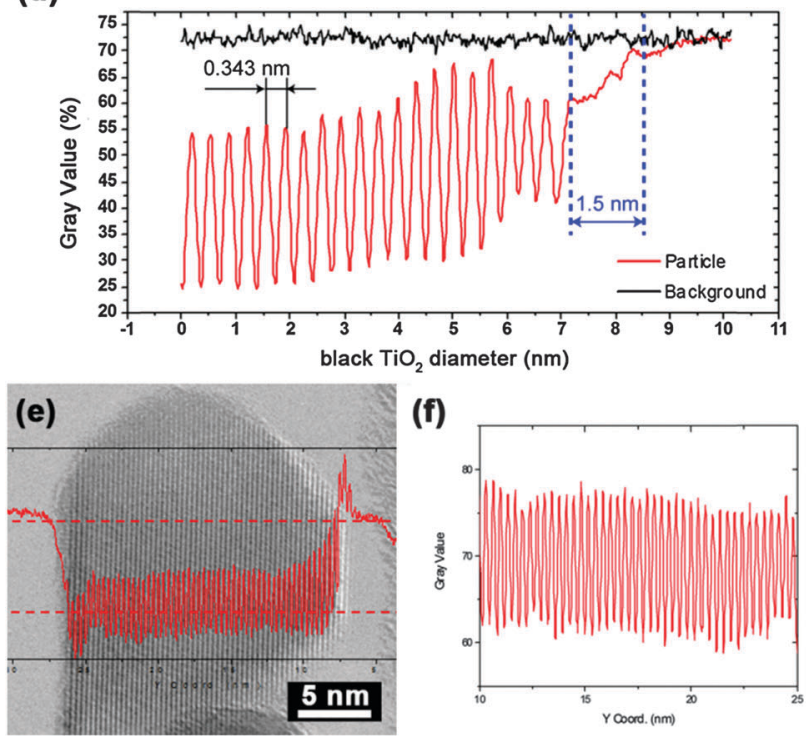

Fig. 1 (a) HRTEM image of a black anatase $\mathrm{TiO}_{2}$ nanocrystal. FFT and reconstructed images of (b) the crystalline core and (c) the boundary area including both the core and the disordered shell. (d) The line profile across the area marked in red in panel (a), showing the lattice distance of the core and the disordered shell thickness taken along the [010] direction. (e) HRTEM micrographs of a P25 nanocrystal with superimposed line profile across all the nanocrystal width. (f) The line profile across the area marked in red in panel (e), showing the lattice distance that characterizes the entire nanocrystal.

crystalline core. $\mathrm{P} 25$, having a phase composition almost identical to that of black $\mathrm{TiO}_{2}$, was chosen as the reference material. ${ }^{21} \mathrm{Fig} .2 \mathrm{c}$ and $\mathrm{g}$ represent the UV-Vis absorption features of the P25 and black $\mathrm{TiO}_{2}$ composites, respectively. Black $\mathrm{TiO}_{2}$ has a strong absorption in the 400-800 $\mathrm{nm}$ region caused by the presence of $\mathrm{V}_{\mathrm{O}} \mathrm{S}$ in its crystalline lattice. The deposition of Pt NPs on P25 did not introduce any new features in the wavelength range investigated herein, while, for black $\mathrm{TiO}_{2}$, light scattering in the visible region notably increased.

Both $\mathrm{Au} / \mathrm{P} 25$ and $\mathrm{Au} /$ black $\mathrm{TiO}_{2}$ show absorption in the 400-500 $\mathrm{nm}$ range related to Au interband transitions occurring at the X-point of the Brillouin zone. ${ }^{5,6}$ On the other hand, the SPR band is due to the collective oscillation of surface electrons in $\mathrm{Au}$ NPs and its characteristics ( $\lambda_{\max }$ and full width at half maximum, i.e., FWHM) are directly related to the NP size distribution. ${ }^{1-4}$ Fig. $2 \mathrm{~d}$ shows the SPR bands having $\lambda_{\max }=$ $548 \mathrm{~nm}$ and $546 \mathrm{~nm}$ (ca. $2.3 \mathrm{eV}$ ) for P25 and black $\mathrm{TiO}_{2}$, respectively. The FWHM appears to be very similar for both samples, pointing out that Au NPs with comparable sizes were 

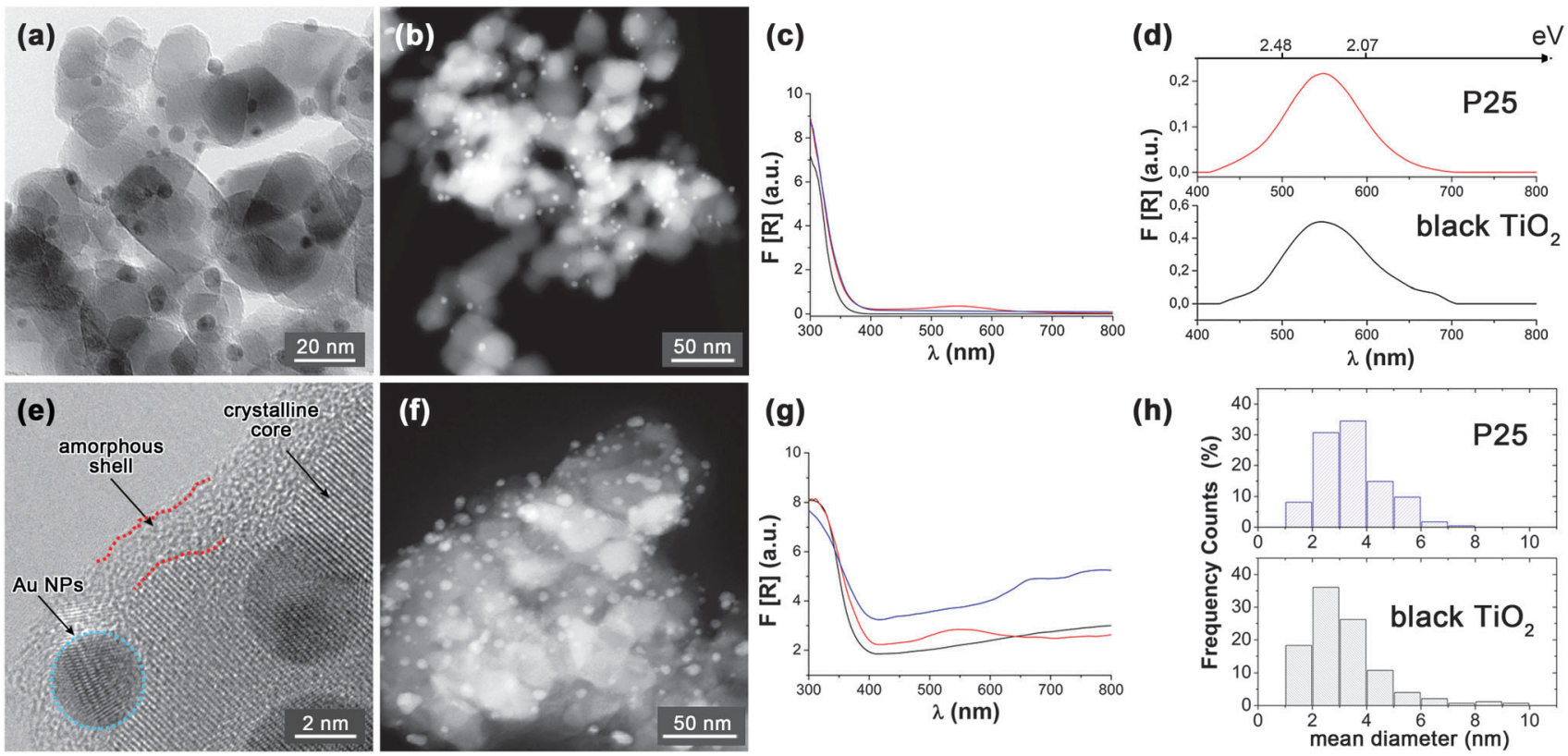

Fig. 2 TEM and STEM images of (a, b) Au/P25 and (e, f) Au/black TiO 2 . Diffuse reflectance UV-vis spectra for (c) P25 (black line), Pt/P25 (blue line), and $\mathrm{Au} / \mathrm{P} 25$ (red line); (g) black $\mathrm{TiO}_{2}$ (black line), Pt/black $\mathrm{TiO}_{2}$ (blue line), and Au/black $\mathrm{TiO}_{2}$ (red line). (d) The SPR absorption band and (h) Au NPs size distribution obtained from frequency counts on STEM images for Au/P25 and Au/black $\mathrm{TiO}_{2}$.

most likely deposited onto both $\mathrm{P} 25$ and black $\mathrm{TiO}_{2}$. The size distributions of Au NPs from STEM analysis (Fig. 2h) are indeed centered at around 3.5 (P25) and $3.2 \mathrm{~nm}$ (black $\mathrm{TiO}_{2}$ ).

The small size of Au NPs ensures a high light absorption/ scattering ratio (scattering becomes predominant for the Au NP size larger than $50-100 \mathrm{~nm}$ ) and a short plasmon dephasing time. ${ }^{1-4}$

Black $\mathrm{TiO}_{2}$ generally exhibits a lower activity in photocatalytic $\mathrm{H}_{2}$ production than $\mathrm{P} 25$-based photocatalysts (see Table S1, ESI $\dagger$ ). This trend can be explained considering the CL spectra of the two supports (Fig. S2, ESI $\dagger$ ). CL spectra of both P25 and black $\mathrm{TiO}_{2}$ consist in a broad band composed of three different components peaking at 2.63, 2.36 (both related to $\mathrm{V}_{\mathrm{O}}$ intragap states) and at $2.77 \mathrm{eV}$, the latter ascribed to the self-trapped exciton. $^{19}$

The higher intensity of $\mathrm{V}_{\mathrm{O}}$-related emissions in the spectrum of black $\mathrm{TiO}_{2}$ directly reflects the higher $\mathrm{V}_{\mathrm{O}}$ s concentration, while the more intense emission of black $\mathrm{TiO}_{2}$ self-trapped exciton is due to its largely distorted structure and to the disordered shell. The location of $\mathrm{V}_{\mathrm{O}}$ in black $\mathrm{TiO}_{2}$ (i.e., localized in the core of the nanocrystals $)^{21}$ plays a crucial role in determining its photocatalytic activity. In fact, the charge carriers generated in the bulk (core) of the semiconductor upon irradiation tend to recombine with high probability because of the large amount of $\mathrm{V}_{\mathrm{O}} \mathrm{s}$. Nevertheless, the carriers that do not thermalize might also experience a strongly reduced mobility through the disordered shell, which likely hinders their diffusion toward the surface. ${ }^{34}$ Such a structural feature produces a discontinuity in the electronic structure of black $\mathrm{TiO}_{2}$, resulting in the formation of an additional interface and in charge recombination occurring at a larger extent with respect to P25. The deposition of Au and Pt NPs on $\mathrm{TiO}_{2}$ samples induced a one order of magnitude enhancement in the rate of photocatalytic $\mathrm{H}_{2}$ production compared to that measured for the bare oxides (Fig. 3(a) for black $\mathrm{TiO}_{2}$ and Fig. S3, ESI $\dagger$ for P25). In any case, hydrogen as well as $\mathrm{CO}_{2}$ and $\mathrm{CO}$ evolution occurred at a constant rate during $\mathrm{H}_{2}$ production, as shown in previous studies. ${ }^{35,36}$

Interestingly, for both supports, the deposition of Au NPs led to higher $\mathrm{H}_{2}$ production rates compared to Pt NPs. These results are in apparent contrast with a recent report showing an opposite trend for stoichiometric monophasic ( $100 \%$ anatase) $\mathrm{TiO}_{2}{ }^{36}$ In this case, the better performance of $\mathrm{Pt} / \mathrm{TiO}_{2}$ was attributed to the greater ability of $\mathrm{Pt}$, with respect to $\mathrm{Au}$, in acting as an electron sink, therefore limiting the electron-hole recombination to a larger extent. ${ }^{36}$

The higher photoactivity of $\mathrm{Au} / \mathrm{TiO}_{2}$ samples observed here can be imputed to different factors, which are, namely, (i) the presence of rutile in both $\mathrm{P} 25$ and black $\mathrm{TiO}_{2}(80 \%$ anatase $20 \%$ rutile); ${ }^{21}$ (ii) a peculiar band alignment due to the presence of oxygen vacancies, (iii) the interaction of gold interband transition with defective $\mathrm{TiO}_{2}{ }^{5}$ and (iv) the possible PIs generated by the interplay between Au NPs and $\mathrm{TiO}_{2}$. TEM analysis provided clear evidence that anatase and rutile clusters grow separately in different black $\mathrm{TiO}_{2}$ crystallites. ${ }^{21} \mathrm{Au}$ NPs with diameters $<5 \mathrm{~nm}$ located at the anatase/rutile interface of $\mathrm{P}_{2} 5 \mathrm{TiO}_{2}$ particles have been already shown to be active sites for plasmonic photocatalysis under visible-light irradiation. ${ }^{7}$ In spite of this, Au/P25 presents only a $5 \%$ increase in the rate of photocatalytic $\mathrm{H}_{2}$ production under full Xe lamp irradiation compared to Pt/P25. In contrast, black $\mathrm{TiO}_{2}$ shows a $33 \%$ enhancement when coupled with $\mathrm{Au}$ NPs rather than Pt NPs. For this reason, considering the $\mathrm{Au} /$ black $\mathrm{TiO}_{2}$ system, the anatase-rutile phase composition cannot be the only factor responsible for the observed photoactivity enhancement. The high concentration of $\mathrm{V}_{\mathrm{O}} \mathrm{S}$ appears to produce a better photoproduced charge stabilization in $\mathrm{Au}$ 


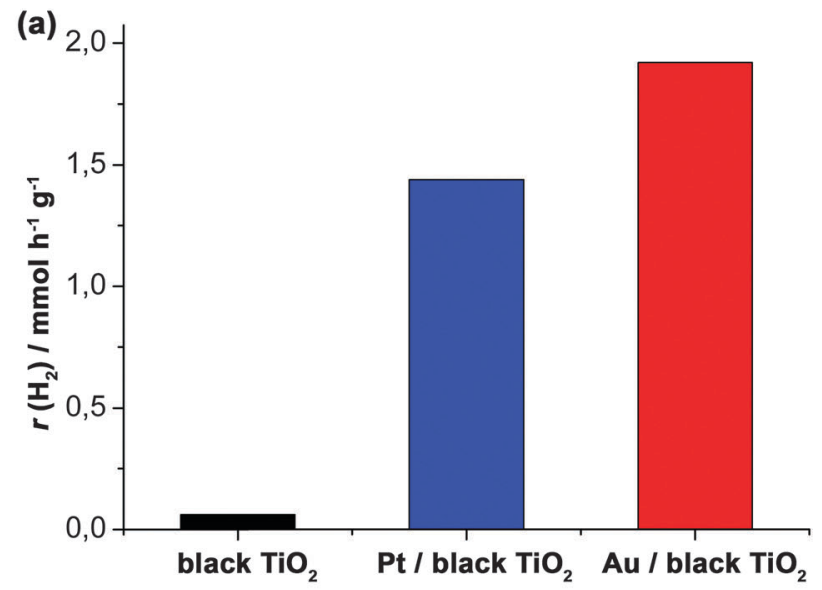

(b)

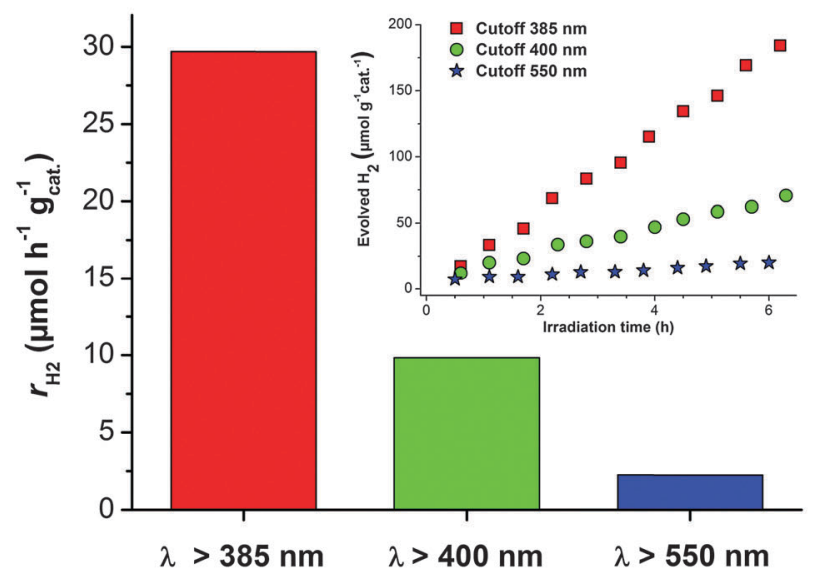

Fig. $3 \mathrm{H}_{2}$ production rates obtained using the black $\mathrm{TiO}_{2}$ photocatalysts in photocatalytic methanol steam reforming under (a) full lamp irradiation, (b) irradiation with different cut off filters: $\lambda>385 \mathrm{~nm}, \lambda>400 \mathrm{~nm}$, and $\lambda>550 \mathrm{~nm}$.

containing photocatalysts accounting for the higher activity measured under full Xe lamp irradiation.

Under these irradiation conditions metal nanoparticles have a dual role: UV light produces $\mathrm{TiO}_{2}$ bandgap excitation promoting the electron flow toward the metal (acting as an electron sink), while visible light induces both $\mathrm{Au}$ interband transitions and plasmon excitation.

In order to better understand the photoresponse enhancement observed in $\mathrm{Au} / \mathrm{black} \mathrm{TiO}_{2}$, photocatalytic hydrogen production experiments were performed employing different cut-off filters (with 385, 400, and $550 \mathrm{~nm}$ cut-off wavelengths) (Fig. 3b). $\mathrm{H}_{2}$ production measured using the $385 \mathrm{~nm}$ cut-off filter is mainly due to bandgap excitation and is poorly informative on the mechanism driven by Au NP excitation. Differently, under irradiation with visible light only (using a $400 \mathrm{~nm}$ cutoff filter), while no $\mathrm{H}_{2}$ was detected when testing bare black $\mathrm{TiO}_{2}$, a $\mathrm{H}_{2}$ production rate of $220 \mu \mathrm{L} \mathrm{h}^{-1} \mathrm{~g}_{\text {cat }}{ }^{-1}$ was measured for $\mathrm{Au} / \mathrm{black} \mathrm{TiO}_{2}$, which is $c a$. $360 \%$ enhancement if compared to the results obtained for $\mathrm{Pt} / \mathrm{black} \mathrm{TiO}_{2}$ under the same experimental conditions (against the 33\% increase observed under unfiltered full lamp light). Under such conditions, bandgap excitation is almost totally suppressed, highlighting the importance of possible $\mathrm{Au}$ interband transitions and plasmonic excitation in photocatalytic $\mathrm{H}_{2}$ production.

By applying a $550 \mathrm{~nm}$ cut-off filter the SPR band was selectively excited, while excluding the more energetic (500-520 nm) interband transitions. ${ }^{5,6}$ The results shown in Fig. $3 \mathrm{~b}$ demonstrate that under these conditions the $\mathrm{H}_{2}$ production was $25 \%$ of that obtained in $400 \mathrm{~nm}$ cut-off filter experiments. The apparent quantum yield was 0.0023 and $0.0016 \%$ in experiments using the $400 \mathrm{~nm}$ and $550 \mathrm{~nm}$ filters, respectively. These findings seem to confirm the existence of interband transitions but still remarks that a certain amount of $\mathrm{H}_{2}$ is produced as a consequence of PIs between Au NPs and black $\mathrm{TiO}_{2}$.

In order to disentangle the interactions between Au NPs and the $\mathrm{TiO}_{2}$ electronic structure that determine the photoactivity trend, we investigated the samples by performing CL spectroscopy. CL spectroscopy is able to electronically excite $\mathrm{Au}$ nanostructures, thus offering a unique tool to investigate their interactions with semiconductors. ${ }^{26,27,37}$ Fig. 4 highlights that the luminescence intensity depends on the deposited metal (either Pt or Au) (see also Table 1), rather than on the support as such ( $\mathrm{P} 25$ and black $\left.\mathrm{TiO}_{2}\right)$. In addition to these general findings, some peculiar features can be appreciated when the CL spectra are deconvolved and more carefully examined. Pt NPs induce a dramatic quenching of the CL intensity over the entire range of

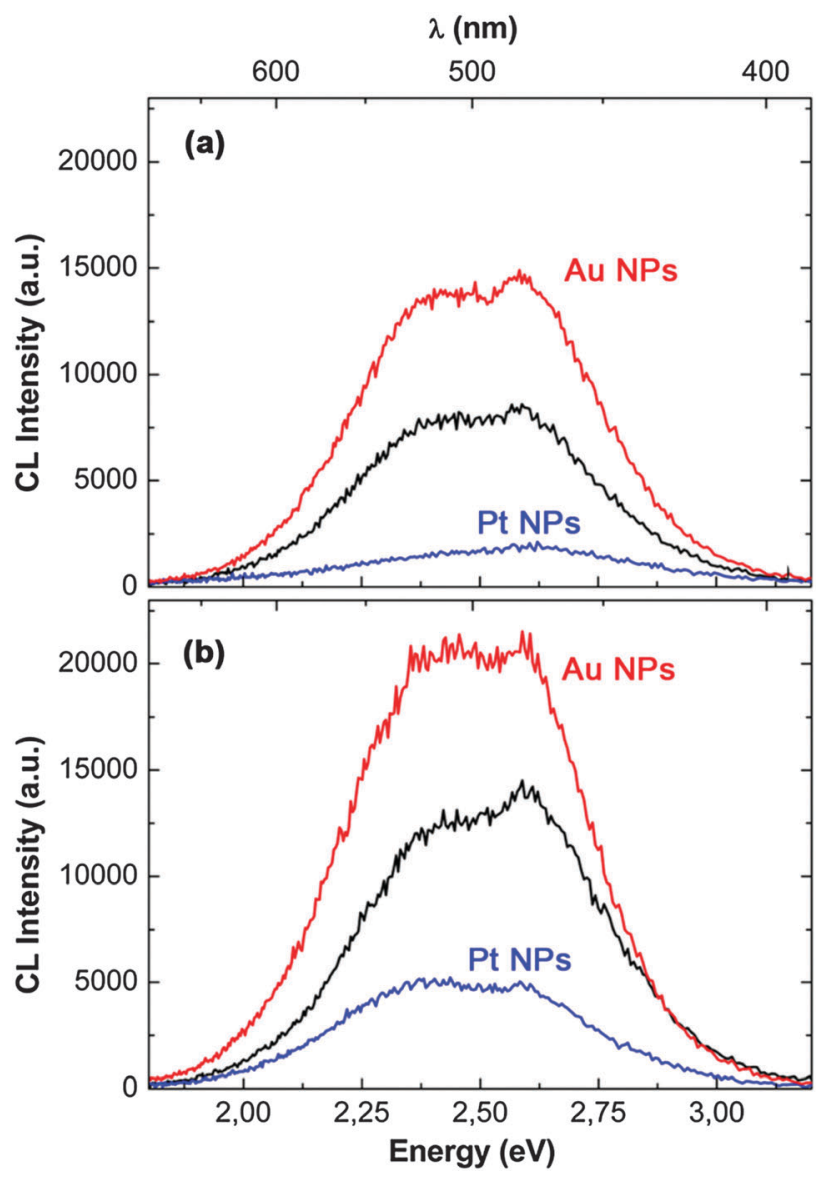

Fig. $4 \mathrm{CL}$ spectra of (a) P25 and (b) blackTiO 2 . Bare $\mathrm{TiO}_{2}$ (black line), $\mathrm{Pt}$ / $\mathrm{TiO}_{2}$ (blue line), and $\mathrm{Au} / \mathrm{TiO}_{2}$ (red line). 
Table 1 Integrated intensity of each components considered in the deconvolution of $\mathrm{CL}$ spectra for black $\mathrm{TiO}_{2}, \mathrm{Au} /$ black $\mathrm{TiO}_{2}, \mathrm{P} 25$, and $\mathrm{Au} / \mathrm{P} 25$

\begin{tabular}{lllrl}
\hline CL component & Black $\mathrm{TiO}_{2}$ & $\mathrm{Au} /$ Black $\mathrm{TiO}_{2}$ & \multicolumn{1}{c}{$\mathrm{P} 25$} & $\mathrm{Au} / \mathrm{P} 25$ \\
\hline $2.77 \mathrm{eV}$ & 1975 & 1383 & 919 & 2253 \\
$2.62 \mathrm{eV}$ & 2224 & 3363 & 1284 & 1500 \\
$2.36 \mathrm{eV}$ & 4071 & 8045 & 2616 & 5200 \\
\hline
\end{tabular}

analyzed energies, while the deposition of Au produces a strong emission enhancement. In the case of Pt, the luminescence quenching is due to the better separation of electron-hole pairs generated by the electron beam. ${ }^{38}$ This behavior closely resembles the results obtained by others using photoluminescence spectroscopy. ${ }^{39}$ Differently, in the case of $\mathrm{Au} / \mathrm{TiO}_{2}$, the enhancement of the CL signals is ascribed to an increased density of charge carriers that recombine through radiative pathways. The increase of the CL intensity consequent to Au deposition (Fig. 4) is related to interactions between the Au-generated carriers and the $\mathrm{TiO}_{2}$ electronic structure. Metal-semiconductor interactions can be classified as: (a) PRET, which would occur between the electromagnetic field, generated by SPR, and the resonant electronic levels of $\mathrm{TiO}_{2}$; (b) hot electron transfer, consisting in the formation of excited electrons that can overcome the Schottky barrier (from interband transitions or SPR) and can be transferred to the $\mathrm{TiO}_{2} \mathrm{CB}$; and (c) thermally excited electron transfer to the $\mathrm{TiO}_{2} \mathrm{CB}{ }^{7-18}$

We rule out pathway (c) since this process, being related to a significant localized heating, was already shown elsewhere to be negligible under experimental conditions similar to those of our experiments. ${ }^{40}$

In order to gain more insights into the role of the electronic levels in CL enhancement (and therefore on photocatalytic $\mathrm{H}_{2}$ production) shown by $\mathrm{Au} / \mathrm{TiO}_{2}$, we consider the integrated CL intensity measured for black $\mathrm{TiO}_{2}$ and $\mathrm{P} 25$, reported in Table 1 (also see deconvolved spectra in Fig. S4, ESI $\dagger$ ). The CL intensity of the self-trapped exciton component $(2.77 \mathrm{eV})$ presents a $75 \%$ increase, when passing from pristine $\mathrm{P} 25$ to $\mathrm{Au} / \mathrm{P} 25$. This can

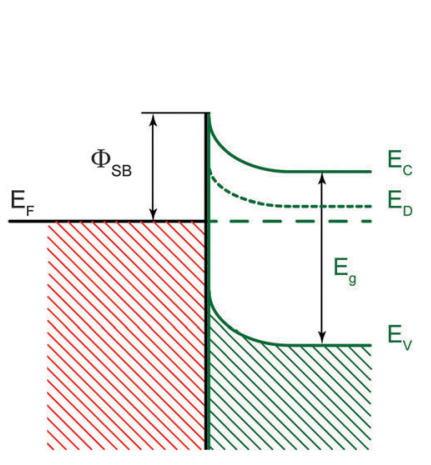

METAL semiconductor

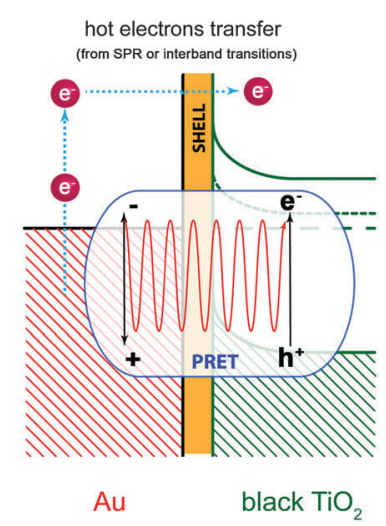

Fig. 5 Schematic representation of the Schottky barrier formation at the n-type semiconductor/metal interface. $E_{C}=$ minimum energy for the $\mathrm{TiO}_{2}$ $\mathrm{CB}, E_{\mathrm{V}}=$ maximum energy for the $\mathrm{TiO}_{2}$ valence band, $E_{\mathrm{D}}=$ donors' (i.e., $V_{O} \mathrm{~S}$ ) energy level, $E_{\mathrm{g}}=$ bandgap, $E_{\mathrm{F}}=$ Fermi level, $\Phi_{\mathrm{SB}}=$ Schottky barrier height. In addition, the scheme shows the transfer of Au hot electrons due to SPR excitation or interband transitions and highlights that the PRET mechanism also occurs for Au/black $\mathrm{TiO}_{2}$. be due to two main mechanisms: (i) the increased charge recombination occurring at the new $\mathrm{Au} / \mathrm{TiO}_{2}$ interface and (ii) the injection of hot electrons from Au NPs into the CB of P25. It is worth noting that a rather different trend is observed for black $\mathrm{TiO}_{2}$ where a modest quenching was recorded.

Interestingly, CL bands related to radiative emission from the $\mathrm{V}_{\mathrm{O}}$ states (peaking at 2.62 and $2.36 \mathrm{eV}$ ) are more intense in the presence of plasmonic Au NPs. These results suggest that intragap electronic states related to $\mathrm{V}_{\mathrm{O}} \mathrm{s}$ (Fig. 5) are directly involved in the transfer of hot electrons that originate mainly, as highlighted by cut-off filter experiments, by interband transitions of Au NPs. Noteworthily, if to a minor extent, excited carriers can also originate from SPR absorption. In addition, an energy transfer by PRET can also be operative since the largest increase of CL emission was recorded at energies corresponding to $\mathrm{V}_{\mathrm{O}}$ states at $2.36 \mathrm{eV}$, i.e., at energies for which the Au NPs exhibit SPR. Therefore, the enhanced visible-light photoresponse of Au/black $\mathrm{TiO}_{2}$ with respect to the Pt-loaded photocatalyst can be imputed both to PRET and hot electron transfer that are able to excite electrons from the valence band to $\mathrm{V}_{\mathrm{O}}$ intragap states and, subsequently, to the $\mathrm{TiO}_{2} \mathrm{CB}$ where they can participate in the $\mathrm{H}_{2}$ evolution reaction. ${ }^{41}$

\section{Conclusions}

In conclusion, we have reported on the use of cathodoluminescence spectroscopy as a unique approach to excite gold electronic transition and study the interactions with intragap state level of semiconductors. In particular, we have investigated composite materials made of $\mathrm{Au}$ and $\mathrm{Pt}$ nanoparticles deposited on the surface of $\mathrm{P} 25 \mathrm{TiO}_{2}$ and core (highly defective)-shell (stoichiometric) black $\mathrm{TiO}_{2}$ nanocrystals.

The peculiar electronic structure and morphological features of black $\mathrm{TiO}_{2}$, i.e. the high oxygen vacancy concentration, allowed us to address and correlate the role of the Au interband and SPR transitions in the photocatalytic production of $\mathrm{H}_{2}$.

Moreover, for the first time, the critical role of intra-bandgap electronic levels of black $\mathrm{TiO}_{2}$ related to the oxygen vacancy in the selective photogenerated charge population due to Au nanoparticles was pointed out.

The described metal-semiconductor interactions involving oxygen vacancies represent a general and innovative criterion that can be exploited in a wide range of technologically relevant applications such as water-splitting devices, solar cells, sensors, and development of analytical tools.

\section{Acknowledgements}

The kind assistance of Dr F. Riboni and Dr M. V. Dozzi in performing photoreforming experiments is gratefully acknowledged. We gratefully acknowledge financial support from the Italian Ministry of Education, University and Research (MIUR) through the FIRB project "Oxides at the nanoscale: multifunctionality and applications" (RBAP115AYN), and from Regione Lombardia through the 
project "TIMES, technology and materials for the efficient use of solar energy" - Accordo Quadro Regione Lombardia - CNR.

\section{Notes and references}

1 S. C. Warren and E. Thimsen, Energy Environ. Sci., 2012, 5, 5133.

2 W. Hou and S. B. Cronin, Adv. Funct. Mater., 2013, 23, 1612.

3 S. Linic, P. Christopher and D. B. Ingram, Nat. Mater., 2011, 10, 911.

4 C. Clavero, Nat. Photonics, 2014, 8, 95.

5 L. Liu, P. Li, B. Adisak, S. Ouyang, N. Umezawa, J. Ye, R. Kodiyath, T. Tanabe, G. V. Ramesh, S. Ueda and H. Abe, J. Mater. Chem. A, 2014, 2, 9875.

6 P. B. Johnson and R. W. Christy, Phys. Rev. B: Solid State, $1972,6,4370$.

7 D. Tsukamoto, Y. Shiraishi, Y. Sugano, S. Ichikawa, S. Tanaka and T. Hirai, J. Am. Chem. Soc., 2012, 134, 6309.

8 J. Lee, S. Mubeen, X. Ji, G. D. Stucky and M. Moskovits, Nano Lett., 2012, 12, 5014.

9 S. Mubeen, J. Lee, N. Singh, S. Krämer, G. D. Stucky and M. Moskovits, Nat. Nanotechnol., 2013, 8, 247.

10 A. Primo, T. Marino, A. Corma, R. Molinari and H. García, J. Am. Chem. Soc., 2011, 133, 6930.

11 A. Tanaka, K. Hashimoto and H. Kominami, J. Am. Chem. Soc., 2012, 134, 14526.

12 H. M. Chen, C. K. Chen, C. J. Chen, L. C. Cheng, P. C. Wu, B. H. Cheng, Y. Z. Ho, M. L. Tseng, Y. Y. Hsu, T. S. Chan, J. F. Lee, R. S. Liu and D. P. Tsai, ACS Nano, 2012, 6, 7362.

13 I. Thomann, B. A. Pinaud, Z. Chen, B. M. Clemens, T. F. Jaramillo and M. L. Brongersma, Nano Lett., 2011, 11, 3440.

14 E. Thimsen, F. Le Formal, M. Grätzel and S. C. Warren, Nano Lett., 2011, 11, 35.

15 H. Gao, C. Liu, H. E. Jeong and P. Yang, ACS Nano, 2012, 6, 234.

16 S. K. Cushing, J. Li, F. Meng, T. R. Senty, S. Suri, M. Zhi, M. Li, A. D. Bristow and N. Wu, J. Am. Chem. Soc., 2012, 134, 15033.

17 Z. Liu, W. Hou, P. Pavaskar, M. Aykol and S. B. Cronin, Nano Lett., 2011, 11, 1111.

18 D. B. Ingram and S. Linic, J. Am. Chem. Soc., 2011, 133, 5202.

19 J. Li, S. K. Cushing, P. Zheng, F. Meng, D. Chu and N. Wu, Nat. Commun., 2013, 4, 2651.

20 X. Pan, M.-Q. Yang, X. Fu, N. Zhang and Y.-J. Xu, Nanoscale, 2013, 5, 3601.

21 A. Naldoni, M. Allieta, S. Santangelo, M. Marelli, F. Fabbri, S. Cappelli, C. L. Bianchi, R. Psaro and V. Dal Santo, J. Am. Chem. Soc., 2012, 134, 7600.
22 A. M. Deml, V. Stevanović, C. L. Muhich, C. B. Musgrave and R. O’Hayre, Energy Environ. Sci., 2014, 7, 1996.

23 J.-Y. Shin, J. H. Joo, D. Samuelis and J. Maier, Chem. Mater., 2012, 24, 543.

24 A. A. Taskin, A. N. Lavrov and Y. Ando, Phys. Rev. B: Condens. Matter Mater. Phys., 2005, 71, 134414.

25 L. Lazzarini, G. Salviati, F. Fabbri, M. Zha, D. Calestani, A. Zappettini, T. Sekiguchi and B. Dierre, ACS Nano, 2009, 3, 3158.

26 E. S. Barnard, T. Coenen, E. J. R. Vesseur, A. Polman and M. L. Brongersma, Nano Lett., 2011, 11, 4265.

27 T. Coenen, E. J. R. Vesseur, A. Polman and A. F. Koenderink, Nano Lett., 2011, 11, 3779.

28 M. V. Dozzi, L. Prati, P. Canton and E. Selli, Phys. Chem. Chem. Phys., 2009, 11, 7171.

29 Q. Hao, B. K. Juluri, Y. B. Zheng, B. Wang, I.-K. Chiang, L. Jensen, V. Crespi, P. C. Eklund and T. J. Huang, J. Phys. Chem. C, 2010, 114, 18059.

30 I. Zorić, M. Zäch, B. Kasemo and C. Langhammer, ACS Nano, 2011, 5, 2535.

31 S. Jung, K. L. Shuford and S. Park, J. Phys. Chem. C, 2011, 115, 19049.

32 C. Langhammer, Z. Yuan, I. Zorić and B. Kasemo, Nano Lett., 2006, 4, 833.

33 C. T. Ko, Y. Y. Han, W. C. Wang, J. Shieh and M.-J. Chen, ACS Appl. Mater. Interfaces, 2014, 6, 4179.

34 S. Kurian, P. Sudhagar, J. Lee, D. Song, W. Cho, S. Lee, Y. S. Kang and H. Jeon, J. Mater. Chem. A, 2013, 1, 4370.

35 G. L. Chiarello, M. H. Aguirre and E. Selli, J. Catal., 2010, 273, 182.

36 A. Naldoni, M. D’Arienzo, M. Altomare, M. Marelli, R. Scotti, F. Morazzoni, E. Selli and V. Dal Santo, Appl. Catal., B, 2013, 130-131, 239.

37 The SPR luminescence is usually detected with an accelerating voltage of $30 \mathrm{keV}$ and then the total impinging power is one order of magnitude higher than in the CL experiments, reported in this work.

38 B. Sun, V. Vorontov and P. G. Smirniotis, Langmuir, 2003, 19, 3151.

39 V. Jovic, Z. H. N. Al-Azri, W.-T. Chen, D. Sun-Waterhouse, H. Idriss and G. I. N. Waterhouse, Top. Catal., 2013, $56,1139$.

40 A. O. Govorov and H. H. Richardson, Nano Today, 2007, 2,30 .

41 G. Barolo, S. Livraghi, M. Chiesa, M. C. Paganini and E. Giamello, J. Phys. Chem. C, 2012, 116, 20887. 